\title{
A Combined Material Substitution and Process Change Approach to Sustainable Batik Production
}

\author{
Abraham Ekow Asmah ${ }^{1}$, Vincentia Okpattah ${ }^{2}$ and Charles Frimpong ${ }^{3 *}$ \\ 1,2Department of Integrated Rural Art and Industry, Kwame Nkrumah University of Science and Technology (KNUST), Ghana \\ ${ }^{3}$ Department of Industrial Art, Kwame Nkrumah University of Science and Technology (KNUST), Ghana
}

Received: January 10, 2018; Published: January 19, 2018

*Corresponding author: Charles Frimpong, Department of Industrial Art, Kwame Nkrumah University of Science and Technology (KNUST), Kumasi

\begin{abstract}
This paper empirically presents a batik production process optimization using sustainable production (SP) methods. The stages of batik production processes involve designing, methodology, dyeing, washing, and finishing. The cost of production is determined by the implementation efficiency and calculation of output to input ratio in the production process. The case study method is employed for production process optimization in two batik enterprises in the Kumasi metropolis. The main objective of this research, is to determine the impact of material sustainability, and the minimization of the production process cost in order to promote maximum operational economic benefits. The implications of this intervention shows that, in batik production process, there could be a saving cost of resisting material by GH\& 31.00 , with a process time of 290 mins and an activity cost reduction of GH\& 68.00 by just replacing paraffin wax with cassava starch. The identified intervention has also been found as significant to address the identified issues that bring a substantial change in the current manufacturing practices of the batik industry. It is recommended that much is to be gained in sustaining the practice when cultural themes are employed in the artifacts created.
\end{abstract}

Keywords: Batik Production; Environmental Resource; Sustainable Production Practices

\section{Introduction}

Batik is a process of dyeing fabric by making use of a resist technique classified by the United Nations Educational, Scientific and Cultural Organization (UNESCO) as part of the "intangible cultural heritage of humanity". It is a traditional creative selfexpressionistic art form in fabric design which originated from the Far East and known throughout the world [1]. In Ghana today, the batik industry is recognized as a profitable enterprise in the fashion industry and is also classified under the small to medium scale creative industry. The potency of this small and medium industry at the local level cannot be over emphasized as its developmental efforts contribute to the quickening of the nation's economic empowerment. Its merchandise is well-known in the creative industries as one of the leading developmental enterprises clustered in Ghanaian communities.

The expectation of the industry is to provide important support to the industrial sector's contribution to local community revenue. However, its production methodology appears to be detrimental to the growth and development of the industry. The cause is not farfetched, in that the prime resist component - paraffin wax - used for its production is a foreign imported material, unsustainable in its supply and harmful to human health due to the heat it generates in the path of the melting and wax application. The cost of production makes its market price much higher than those from foreign lands. Comparatively, the cheapness of foreign batiks and the high selling price of locally produced batik compete unfavorably in the Ghanaian market. To avert this trend there is the urgent need for the implementation of sustainable production practices that offers an advantage for the progress and development of the batik industry.

This is premised on the fact that using cassava starch and its modification for batik making in Ghana due to its accessibility and sustainability is far more beneficial than the traditional use of the exportable paraffin wax which sometimes is unavailable and yet expensive in the marketplace. This makes the end product very expensive to the low income earner. Cassava offers a broad scope of applications with its overall benefits and provides livelihood to most farmers in the rural areas. Cassava cultivation is practiced in all the Agro-ecological zones in Ghana which gives strength to its sustainability in terms of cultivation [2]. In Ghana, it is estimated that $70 \%$ of farmers grow cassava, contributing $16 \%$ of Ghana's 
Agricultural Gross Domestic Product (AGDP) according to SafoKantanka [3] and consumed by more than $80 \%$ of the population $[3,4]$. The cassava flours used for this innovative batik print are of two types, the white powdered flour starch used for laundry finishing treatment and the brown powdered flour starch used for food [5].

Thus far, the technique employed in Nigeria and elsewhere in the production of Adire is the white cooked starch as Braide and Adetoro [6] describe. The method of implementation is either by free-hand painting with the cooked cassava starch, or by stenciling the starch on the material. The stencils are made with corrugated zinc or a perforated tin sheet which allows the starch print on the fabric. Kadolph [7] advocates the use of cooked starch paste in Nigeria via the screen print method to force the paste out of the screen onto the fabric with the squeegee to produce Adire. Picton and Mack [8] also affirm the use of cooked cassava starch mixed with a small amount of copper sulphate to produce the Adire Eleko resist dyed fabric using the same procedure mentioned above.

Important materials commonly used as binders for traditional textiles are starch, derived from tree gums (acacia), food grains, stems or bases such as maize, wheat, tapioca and cassava. The choice of cassava starch as the vehicle for sustainable production practices is as a result of the following characteristics when applied in textile printing. Its high molecular weight compounds give a better viscous paste in water below $90^{\circ} \mathrm{C}$ since hot starch pastes continue to lose viscosity if maintained near boiling temperatures [9]. They impart adhesiveness and softness to the printing paste when used on a fabric surface without spreading. They are capable of maintaining the design outlines even under extreme pressure. They hold on to the dye particles in the desired area on the fabric until the transfer and subsequent fixation of dye onto the fabric. Its viscosity is sufficiently high to prevent rapid diffusion of the colour through the fabric and to avoid poor print definition outlines. It delivers a stable paste viscosity, which grants an even and measured flow through the sieve. The viscosity stability is not only durable in terms of the time during which the cloth is being printed, but even through the dyeing operation [10]. The resultant cohesiveness ensures that the forcefulness and the free flowing quality of the paste remains intact after its formulation and execution.

\section{Disadvantages of Paraffin Wax}

Burning from paraffin wax emit carcinogens into the air. This high temperature fast-burning wax creates a plethora of byproducts, including benzene, acetone, xylene, formaldehyde and acetaldehyde [11]. Paraffin wax as stated above has some limitations;

a. Their application process generates hazardous chemicals, creating worker safety concerns,

b. They may generate hazardous wastes, and

c. These waxes are not environmentally friendly. The soot created from these wax has been known to cause asthma and allergy flare-ups [11]. Paraffin wax is more expensive than regular cassava starch. Removing paraffin wax from the dyed cloth uses a difficult dewaxing process that requires hot boiling water and even much disturbing when bee wax is used which requires an addition of soap.

Cassava like starch on the other hand can be washed away with just cold water. Hot paraffin wax in use is very hot and thus devastating when it gets in contact with human skin. Cassava starch however is applied virtually in a cold state. "Ice patterns", usually referred to as vein lines created when wax cools on fabric and the dye liquor penetrates, is perhaps a significant advantage of using paraffin wax over the use of starch. Its operational residue on the floor coming in contact with heat or the sun's ray becomes a danger to workers due to its slippery nature.

\section{Advantages of Cassava Starch}

The natural thickening agent (cassava starch) used for the print paste is available and easily accessible to traditional batik and tie-dye printers all over the country. They impart adhesiveness and softness to the printing paste so it can be applied to a fabric surface without spreading. They are capable of maintaining the design outlines even under excessive pressure. They adhere to the dye particles in the desired place on the fabric until the transfer of the dye into the fabric and its fixation are complete. Its viscosity is sufficiently high to prevent rapid diffusion of the colour through the fabric, in order to avoid poor print definition outlines [12]. It has a stable paste viscosity, which allows an even and measured flow through the screen. The viscosity stability is not only durable in terms of the time during which the fabric is being printed, but even through the dyeing process, [10]. The resultant cohesiveness ensures that the strength and the free flowing quality of the paste remains intact even after its preparation and its execution [13].

Preparation of starch is much slower and at a cooler temperature than does paraffin wax. It is a process that emits less soot, if any, than paraffin wax and therefore environmental toxicity with implications on human health is eliminated. Because the starch is locally made, every purchase of it boosts the starch market and helps support farmers. Creating intricate design patterns with starch is easier to control than paraffin wax due to its cooler temperature and slower fluidity when in use than paraffin wax which tends to work better when hot with low viscosity making it difficult to control or require a higher expertise to control. Perhaps the greatest advantage in the use of starch as a resist medium is the whole change in technique that employs only a screen and squeegee.

Wax application as resist medium however, requires the use of wax application tools to print the design. The starch application technique, with its unique properties, has the ability to mimic any design and produce diverse textural effects on any substrate with high artistic value. The study, therefore, unveils the results of a pilot project that explored an abounding environmentally degradable organic material (cassava starch) as a vehicle and a resist medium in the process of batik production thus effectively reducing the production process time. This was made possible by the varied abilities of cassava to be transformed into different starch types, modified in its preparation to attain varied consistency and 
characteristics at different temperatures during preparation and application. The inference drawn is that the new methodology adapted is able to replace the melted wax effectively and yet maintain the batik characteristics with an enhanced well-defined images and clarity of colour prints at the end of the printing operation. This unique production methodology not only extends opportunities for adult creative work, but also makes room for kids from age 4 upwards with little help.

\section{Methodology}

In this study, a sustainable production method is used to optimize the production process of batik in two prominent batik enterprises in Ashanti Region of Ghana used for the pilot project. The main objective of this optimization is to minimize the costs incurred in the production process of batik in order to obtain maximum benefit. The innovative production method was designed to optimize the production goals of Asfatex enterprise and Craft Consult by using cassava starch and paraffin wax and other common limits of existing resources in their operations. The two basic materials in contention used are the paraffin wax and the starch and its modifications as resist mediums. A mixed methodology comprising case study, experimental and exploratory methods under the qualitative research approach was used. The experimental mainly employed the practice based research that depended on studio activities. The studio experiment contributed to the analysis established in the new sustainable production approach to create the unique type of dye prints [12]. Results of experiments conducted carefully recorded, analyzed and interpreted.

The qualitative research gave a holistic picture of what goes on in the dyeing industry [14]. The purposive sampling technique was used in selecting both the materials and the enterprises for the study, with interviews being a major tool for collecting data that was consequently authenticated by means of triangulation. The respondents were chosen based on their technical knowledge and batik-centered businesses and comprised home-based batik craft artisans, small scale batik enterprises, and consumers. There were 8 hand-drawn batik enterprises, 10 stamped batik enterprises, 2 printed batik enterprises, 7 batik garment manufacturers, 6 knowledgeable academicians, and 13 households-managed business units of batik trade.

These enterprises were used to ascertain their acceptance or rejection of starch as a substitute for paraffin wax in the manufacturing of batik. Since competition is no longer between enterprises, but among sustainable manufacturing practicing firms, effective, sustainable manufacturing practices assume a potentially valuable way of securing competitive advantage and improving industrial performance. This research conceptualizes and developed three dimensions of sustainable manufacturing practices which are the effective utilization of environmental material product and the culturally sustainable production practices and that of the manufacturing product. The anticipated benefits of sustainability though considered as latent variables was to:

\section{A. Minimise health implications}

B. Maximize financial benefits

C. And promote cultural communication that will lead to trade opportunities. The parameters used for the pilot experiments in its computations considered the use of water resources, the use of the resist medium, the consumption of the energy resources and its cultural sustainability.

\section{Materials}

Aside the main resist materials mentioned in the review, the following common materials were used in the project; $100 \%$ mercerized cotton, tracing paper, powdered cassava starch, vat dye, distilled water for recipe preparation, aluminium/stainless pots, plastic palette bowls, cups and spoons, big plastic bowls as dyebaths. Others included small plastic palette bowls for measuring dyes, wax, mild detergent, heat source (coal pots), metal bucket, rubber gloves, thumb-tacks, P.V.A (Carpenters glue), Potassium dichromate, sodium hydrosulfite (Na2S204), sodium hydroxide $(\mathrm{NaOH})$ ), wooden ladle and aprons. Tools and equipment employed included, staple machine and pins, a pair of scissors, cutting tool, coating trough, squeegee, mesh, cello-tape, pens and pencils. A ruler, tjanting, masking tape, pressing iron, working table, and working shed, camera, computer, Wooden frames, and water reservoir for washing were additionally used. Corresponding differences in material and tool usage that were considered in the project were as follows; paraffin wax to replace cassava starch and vice versa, wooden designed stamps to replace silk designed screens

\section{Results and Discussion}

\section{A Proposed Production Model}

Based on the comprehensive study of the batik making process, a new production model was proposed with the aim of minimizing cost by utilizing an available environmentally friendly material like the local starch, to conserve energy. The new sustainable manufacturing model aims at integrating culturally sustainable approach to product design, with new application tools and methodologies in every single step of production. In fact, the case study of enterprises mentioned showed that the implementation of this new paradigm could lead to new market opportunities for the batik industry. Findings suggest that different types of environmentally sustainable manufacturing practices associated with different competitive outcomes may emerge. These specific findings could be helpful to textile designers and practitioners as they respond to environmental and competitive demands.

Though sustainable manufacturing is widely regarded as a business strategy, few researchers have concentrated on the validation of its positive link with business performance [15]. The proposed hypotheses have been tested to empirically validate the proposed model by means of conducting a summation survey among the 2 small enterprises (SMEs) located in Kumasi. The research hypotheses considered in the study indicated that the utilization of environmental resources, to promote economic 
benefits, and cultural sustainability is feasible in batik production. However, no previous study had tried to propose the possibility of utilizing environmental resources, to promote economic benefits, and the environmental sustainability of batik production in Ghana.

Table 1 shows the current key raw materials, material cost, process time and cost of activity in batik production process used to produce batik cloth. Table 2 shows the raw material, material cost, process time and cost of activity in batik production process used in the intervention model to produce batik. Cost of materials is stated in Ghana cedis, which indicates the amount of cost needed for the procurement of raw materials to produce 6 batik cloths for each model. The processing time is expressed in hours/minutes, which shows the amount of time required for the production process of 6 metres of batik cloth. The cost of an activity is indicated per work done at each stage of production for the 6 yards fabric and not necessarily the cost of production per month of each worker.

\section{Traditional Manufacturing Practices}

The entire production process adopted the current traditional practices that batik practitioners are familiar with using the coal pot and charcoal as a source of energy. The process engaged non-consumables and consumables but concentrated on the consumables and function driven activities since the two models all used the same non-consumables for the projects. The duration of the production process was between an hour and 2 days. The production process was executed by 2 employees from each enterprise. The production costs were calculated without taking their salaries into account. Tables $1 \& 2$ recorded the production process time for batik and the cost of raw materials needed as GHI 290.00 and GHI 259.00 with as much as 520 and 230 mins of production time respectively. The cost of activity for Table 1 was GHI160.00 as against GH 68.00 for (Table 2). The savings accrued for material cost was therefore realized.

Table 1: Key Material Cost, Process Time and Cost of activity in Batik Production Process for traditional Batik manufacturing processes.

\begin{tabular}{|c|c|c|c|c|c|}
\hline Design process & Material. & Material Quantity & $\begin{array}{l}\text { Cost of material. } \\
\text { (GHI - Cedes) }\end{array}$ & Process Time (mins) & Cost of activity. \\
\hline Drawing or sketching & Pencil or pen. & 1 & 1 & 5 mins & \\
\hline \multirow{5}{*}{$\begin{array}{l}\text { Construction of tools } \\
\text { for melting of wax }\end{array}$} & Paraffin wax & $1 / 2$ bar & 35 & & \multirow{5}{*}{140} \\
\hline & Melting pans & 1 & 15 & & \\
\hline & Tjanting Tool (foam) & 1 & 1 & 240 mins & \\
\hline & Wooden Stamp & 2 & 30 & & \\
\hline & Foam Stamp & 2 & 10 & & \\
\hline \multirow{6}{*}{ Items \& Dye Mixing } & Sodium Hydroxide & 1 sachet & 3 & & \\
\hline & Sodium Hydrosulfide & 2 sachets & 4 & & \multirow{5}{*}{1} \\
\hline & Vat Dyes & 3 sachets & 9 & $45 \mathrm{mins}$ & \\
\hline & Dyeing Bowls & 3 & 45 & & \\
\hline & Mixing Pallet Plastic & 3 & 15 & & \\
\hline & Plastic Spoons & 3 & 1 & & \\
\hline $\begin{array}{l}\text { Substrate decoration } \\
\text { from Printing to } \\
\text { Dyeing }\end{array}$ & Mercerized cotton & 6 metres & 24 & 150 mins & 12 \\
\hline \multirow{3}{*}{ Means of Protection } & Aprons & 1 & 15 & & \\
\hline & Surgical Gloves & 1 box & 20 & & \\
\hline & Nose \& Mouth Guard & $1 \mathrm{set}$ & 15 & & \\
\hline Energy application & Heat device(charcoal) & $1 / 4$ bag & 10 & & \\
\hline Processing solvent & Water & 10 buckets & 3 & & \\
\hline \multirow{2}{*}{ De- waxing } & Bucket of boiling water & 1 & 10 & 60 mins & \multirow{2}{*}{5} \\
\hline & Wooden spatula & 1 & 10 & & \\
\hline \multirow{2}{*}{ Washing process } & Washing bowl & 1 & 15 & 20 mins & \multirow{2}{*}{2} \\
\hline & Washing Detergent & 1 & 2 & & \\
\hline Grand Total & & & 290 & 520 mins & 160 \\
\hline
\end{tabular}


Table 2: Key Material Cost, Process Time and Cost of activity in Batik Production Process for the Proposed Intervention.

\begin{tabular}{|c|c|c|c|c|c|}
\hline Design process & Material application. & Material Quantity & $\begin{array}{l}\text { Cost of material. } \\
\text { (GHI - Cedes) }\end{array}$ & Process Time (mins) & Cost of activity. \\
\hline Drawing or sketching & Pencil or pen. & 1 & 1 & 5 mins & \\
\hline \multirow{5}{*}{ Screen development } & Lacquered screen & $70 \mathrm{ml}$ & 10 & \multirow{5}{*}{180 mins } & \multirow{5}{*}{80} \\
\hline & Potassium di-chromate & 3 spoons & 4 & & \\
\hline & PVA & $70 \mathrm{ml}$ & 15 & & \\
\hline & Wooden frame & 3 & 75 & & \\
\hline & Silk mesh & 3 metres & 40 & & \\
\hline \multirow{6}{*}{ Dye Mixing } & Sodium Hydroxide & 1 sachet & 3 & \multirow{6}{*}{10 mins } & \multirow{6}{*}{1} \\
\hline & Sodium Hydrosulfide & 2 sachets & 4 & & \\
\hline & Vat Dyes & 3 sachets & 9 & & \\
\hline & Mixing pallet Bowls & 3 & 15 & & \\
\hline & Powdered starch & 3 cubes & 5 & & \\
\hline & Plastic Spoons & 3 & 1 & & \\
\hline \multirow{2}{*}{ Substrate decoration } & Fabric for printing & 6 metres & 24 & \multirow{2}{*}{30 mins } & \multirow{2}{*}{10} \\
\hline & Konkonte for printing & 2 table $s p$ & 2 & & \\
\hline \multirow{3}{*}{ Means of Protection } & Aprons & 1 & 10 & & \\
\hline & Surgical Gloves & 1 box & 20 & & \\
\hline & Nose \& Mouth Guard & $1 \mathrm{set}$ & 15 & & \\
\hline Energy application & Heat device (charcoal) & $1 / 16$ bag & 2 & & \\
\hline Processing solvent & Water & 6 buckets & 2 & & \\
\hline \multirow{2}{*}{ Washing process } & Washing Bowl & 1 & 5 & \multirow{2}{*}{5 mins } & \multirow{2}{*}{1} \\
\hline & Detergent & 1 & 2 & & \\
\hline \multicolumn{3}{|c|}{ Grand Total } & 259 & 230 mins & 92 \\
\hline & Accrued savings & & 31 & 290 mins & 68 \\
\hline
\end{tabular}

A critical factor worth mentioning was the fact that the chemical content within the various pastes formulated for Table 2 , required a lacquered screen to secure its effectiveness of accommodating the various formulated print pastes. This was extremely important to avoid the chemical corrosion of the photographic film of the screen during the printing process [12]. To commence, four types of screens were developed,

a) Three of them was to transfer the varied dye paste to produce the printed fabrics and

b) The last, was to allow the resist konkonte paste to cover the already printed portions of the fabric, leaving the unprinted portions uncovered to allow the intake of the dye during dyeing by immersion.

Unlike the traditional batik printing process, all the three colors were printed one after the other, before the final konkonte covering and dyeing by immersion. Traditional dyeing however, followed a series of waxing, dyeing, re-waxing and re-dyeing of colour dyes within a two day period to achieve almost the same results. Thus, the differential materials used are the cassava starch and the silk screen instead of wax and wooden block. The outcome of the process is an improved quality, alongside improved efficiency in production, time management and waste minimization, an envisaged increase in market share, creation of new markets, lower costs, innovation, and improved flexibility, which are all key performance criteria for industries engaged in sustainable practices. The implication of this intervention shows that use of the locally available raw material saves cost by GH\& 31.00, with a process time of $290 \mathrm{mins}$ and an activity cost reduction of GH\# 68.00. Additionally, operational cost was reduced, resulting in improved profitability, worker safety and the advantage of using an environmentally-friendly product and minimizing environmental pollution.

The efficient use of cassava starch obviously ensures that manufacturing activity are sustained due to their availability. Having dealt with the main material that impedes safety in batik manufacturing, the youth are likely to be encouraged to engage with the industry to promote sustainable rural and urban development. The implication of this intervention potentially offers the possibility of marketing its products to both local and international markets as it finds expression in traditional clothes and garments to government and private organizations. Its patronage will even increase if their aesthetic characteristics reflect the Ghanaian identity or are the expression of the people's culture through cherished traditional apparels. This effort made to revitalize batik making is an important driver for sustainable rural development because any economy built on an available natural resource base has the likelihood of improving the vocational sector of the economy [16]. The youth equipped with such skill in schools will 
also strengthen the concept of made in Ghana goods to facilitate the patronage, promotion and the sustainability of locally made textiles. Holistic Economic Development based on technological innovation processes and natural resource exploitation is believed to be the key to the future of the batik industry.

\section{Conclusion}

This paper examined the sustainability of batik production and its material development strategies in Ghana. It suggests that the batik enterprises are important in economic and social terms, in the short-run by providing incomes, jobs, especially for artisans, and foreign currency receipts and in the long run by providing the country the opportunity for sustained economic development through appropriate technology to enhance the dynamic effects of batik clothing. The potential of its raw materials and methodology to contribute to long-run growth and sustainable development depend not only on the qualities (desirable or otherwise) of the product, but also on the quality and effectiveness of production processes and availability of raw materials at a low cost within the country's forest and market space. The effects on employment of the batik enterprises are quite significant for economic development compared to the overall share of the country's total employment in the cloth manufacturing sector within the various communities in Ghana.

An important factor for the sustainability of an enterprise is the genuine involvement of the local people (farmers) as active participants whose beneficial concerns and experience are essential to the enterprise's success [17]. The level of community support determines whether the enterprise becomes successfully established, and how it quickly responds and adapts to meet changing needs. By exploiting Ghana's low labour cost advantages to fill emerging niches and meet buyer demands, its dynamic effects for sustainability becomes even greater, if more linkages can be built up between the production centres and the community. In effect, the characteristics of the batik product (relatively low capital intensity; low investment costs; and use of low skilled labour), also mean that the industry is relatively footloose and able to adjust to changing market conditions quickly and further increasing its sustainability [18].

Though batik making is seen as a productive venture with export potential, it lacks fast production approaches to meet higher consumer demands and therefore needs to be revived and sustained as an industry. Making room for new developmental ideas will inject new techniques of production and make production easier and sustainable. The selection of right material is critical, as it does not only affect the product's cost, enhance methodology, quality, and growth of batik enterprises, but also impact the environment. Adopting such innovative practices will guarantee the benefits derived in the environment, to promote safety, minimize energy utilization and health of producers [19].

\section{Recommendations}

It is highly recommended that batik be introduced into a broader public space to provide a viable platform for engagement between academia and industry through studio base learning (workshops) of batik design processes not only in educational institutions, but also in batik making communities. The focus of sustainability and development in this direction needs to be strengthened using different local material components and design methodologies that relate to traditional African textile culture. Finally, for sustainability of the batik craft to be successful, it is also recommended that skills training of batik producers be intensified and encouraged to create new forms using varied methodologies.

\section{Acknowledgement}

We would like to acknowledge Miss Juliet and Miss Christina Mensah for their contribution to the background work and critical comment on the paper. The authors would like to thank the following for their support of this project: Asfatex enterprise in KNUST campus and craft consult at Bomso

\section{References}

1. Akhir NHM, Ismail NW, Said R, Kaliappan SR (2016) Creative Craft: The Uniqueness and Potential of the Malaysian Batik Industry.

2. Graffham AJ, Ababio JT, Dziedozoave N, Day G, Andah A, et al. (1998) Market potential for cassava flours and starches in Ghana. Tropical agriculture-london then trinidad 75: 267-270.

3. Safo KO (2004) Colocasia esculenta (L) Schott. Plant Resources of Tropical Africa 2: 206-211.

4. Parkes E (2009) Using molecular markers to identify new sources of CMD resistant genes for cassava improvement. In $2^{\text {nd }}$ GSS Workshop CIAT Cali Colombia, USA.

5. Taylor N, Chavarriaga P, Raemakers K, Siritunga D, Zhang P (2004) Development and application of transgenic technologies in cassava. Plant molecular biology 56(4): 671-688.

6. Braide 00, Adetoro SA (2013) Cassava flour as a resin printing paste for textile patterns, Abeokuta, Nigeria. Transnational Journal of Science and Technology 3(8): 1-59.

7. Kadolph SJ (2007) Quality assurance for textiles and apparel. Fairchild Publications, UK.

8. Picton J, Mack J (1989) African textiles. Trustees of the British Museum, USA.

9. Lund D, Lorenz KJ (1984) Influence of time, temperature, moisture, ingredients, and processing conditions on starch gelatinization. Critical Reviews in Food Science \& Nutrition 20(4): 249-273.

10. Pfaff G (2008) Special effect pigments: technical basics and applications. Vincentz Network GmbH \& Co KG.

11. Denise K (2012) Advantages and Disadvantages of Soy \& Paraffin Candles.

12. Asmah AE, Okpattah V, Frimpong C (2015) Kanto: An Innovative Approach to Batik Production. International Journal of Innovative Research and Advance Studies 2(1).

13. Radley JA (1976) Industrial uses of starch and its derivatives. Applied Science Publishers, London p. 26-28.

14. Frankel RM, Devers K (2000) Qualitative research: A consumer's guide. Educ Health (Abingdon) 13(1): 113-123.

15. Jayachandran R, Singh S, Goodyer J, Popplewell K (2006) The design of a sustainable manufacturing system: A case study of its importance to product variety manufacturing. In $2^{\text {nd }}$ Virtual International Conference on Intelligent Production Machines and Systems pp. 650-655. 
16. Fernandez R, Rogerson R (1995) On the political economy of education subsidies. The Review of Economic Studies 62(2): 249-262.

17. Admassu M, Kumie A, Fantahun M (2003) Sustainability of Drinking Water Supply Projects in Rural of North Gondar, Ethiopia. Ethiopian Journal of Health Development 17(3): 221-229.
18. Nordås HK (2004) The global textile and clothing industry post the agreement on textiles and clothing. World $7(1,000)$.

19. Zhou CC, Yin GF, Hu XB (2009) Multi-objective optimization of material selection for sustainable products: artificial neural networks and genetic algorithm approach. Materials \& Design 30(4): 1209-1215.

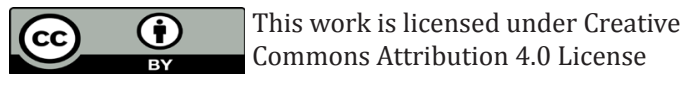

DOI: 10.32474/LTTFD.2018.01.000104

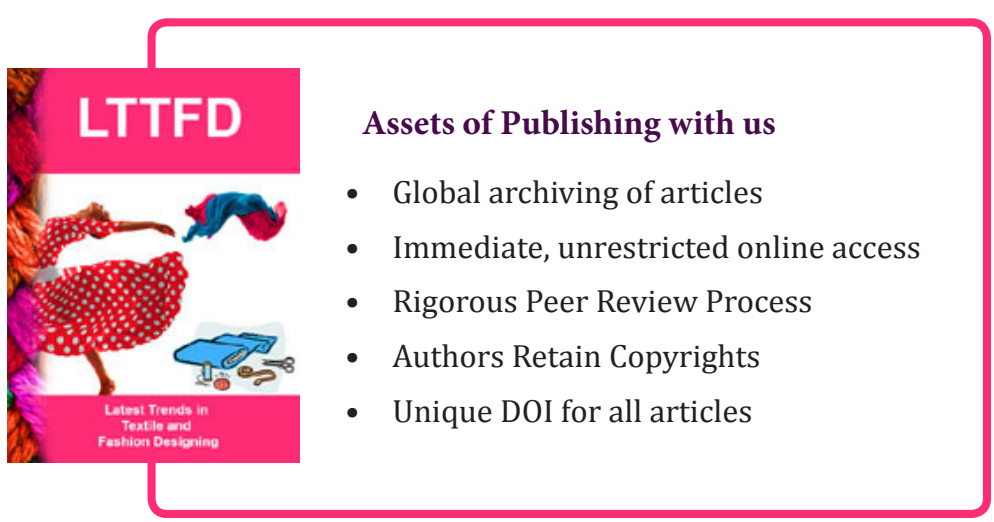

